\title{
Audiological profile in fitness instructors
}

\author{
D Felícia ${ }^{1}$, c Nazaré ${ }^{1}$ \\ ${ }^{1}$ ESTeSC - Coimbra Health School, Instituto Politécnico de Coimbra, \\ Portugal \\ Introduction:
}

Hearing loss due to exposure to noise in the workplace is a significant public health problem. Studies indicate that more than half of fitness instructors have signs or symptoms of hearing loss (that interfere with their quality of life) due to exposure during fitness classes to music with high intensity levels and/or for long periods of exposition (exceeding the limits of occupational health and safety, and at risk of developing hearing loss) and due to the fact that they don't wear hearing protection during classes and because gyms most often do not present adequate acoustic environment.

\section{Objectives:}

To evaluate the auditory system of fitness instructors exposed to high intensity levels of music during fitness classes, in order to increase fitness instructors awareness to prevent hearing loss.

\section{Methods:}

There were compared 2 groups: 15 fitness instructors and 25 subjects from control group (21-38 years) through auditory thresholds (pure-tone audiometry from 0.125 to $8 \mathrm{kHz}$ and high frequencies from 9 to $12.5 \mathrm{kHz}$ ) and cochlear function (distortion products otoacoustic emissions from 0.5 to $10 \mathrm{kHz})$.

\section{Results:}

The auditory thresholds mean in both ears was higher in fitness instructors than in the control group for all frequencies in pure-tone audiometry and high frequencies, with statistically significant differences and the amplitude mean of the otoacoustic emissions was lower in instructors at several frequencies in both ears.

\section{Conclusions:}

The results show these fitness instructors had an increase in auditory threshold and a decrease in otoacoustic emissions amplitudes compared to the control, which indicates that the music presented in some gyms during the fitness classes is having negative consequences for the auditory system. These findings cannot be neglected. Fitness instructors should be aware of the risks of exposure to music for their hearing, when exposure exceeds recommendations, and a periodic auditory monitoring and protection are advised. 